\title{
LA SALUD OCUPACIONAL DE UNOS ADULTOS MAYORES EN UN CONTEXTO ELECTORAL DE UNA LOCALIDAD DEL CENTRO DE MÉXICO
}

\author{
Cruz García Lirios ${ }^{1}$, Javier Carreón Guillén ${ }^{2}$ y José Marcos Bustos Aguayo. ${ }^{3}$
}

Resumen. El objetivo del presente trabajo fue dilucidar los significados en torno a las categorías de agenda pública de salud, posicionamiento y procesualismo con la finalidad de interpretar los discursos de grupos excluidos. Se llevó a cabo un estudio no experimental, exploratorio, transversal y cualitativo con un muestreo no probabilístico de siete informantes, considerando estatus económico, laboral, civil y social. Los resultados muestran que las categorías en comento legitiman las diferencias entre la salud ocupacional de la juventud con respecto a la vejez, así como el papel del Estado como administrador de fondos de retiro o apoyos económicos. Se advierten líneas de investigaciones empíricas y contrastables.

Palabras clave: salud, juventud, vejez, pensión, discurso

\section{THE OCCUPATIONAL HEALTH OF SENIOR ADULTS IN AN ELECTORAL CONTEXT OF A LOCALITY IN THE CENTER OF MEXICO}

Abstract. The objective of this paper was to elucidate the meanings around the categories of agenda, positioning and processualism in order to interpret the discourses of excluded groups. A non-experimental, exploratory, cross-sectional and qualitative study was carried out with a non-probabilistic sampling of seven informants, considering economic, labor, civil and social status. The results show that the categories in question legitimize the differences between the occupational health of the youth with respect to old age, as well as the role of the State as administrator of

\footnotetext{
${ }^{1}$ Realizó estudios de doctorado en Psicología Social y maestría en Trabajo Social, Universidad Nacional Autónoma de México. Es licenciado en Psicología Social por la Universidad Autónoma Metropolitana. Profesor de asignatura en el Instituto Politécnico Nacional, Universidad Autónoma del Estado de México y Universidad Autónoma del Estado de Morelos. Línea de investigación en "Gobernanza de la Educación y Salud Pública en Organizaciones de la Sociedad Civil”. página web: https://www.researchgate.net/profile/Cruz_Lirios Teléfono: (52) 5556226666 ext 47385 correo electrónico: cgarcial213@uaemex.mx

${ }^{2}$ Doctor en Administración por la Facultad de Contaduría y Administración de la Universidad Nacional Autónoma de México. Profesor de Carrera en la Escuela Nacional de Trabajo Social de la UNAM, y adscrito al Sistema Nacional de Investigadores. Línea de investigación: "Encuadre de la prensa en torno a la violencia sociopolítica en México. Análisis transcultural de la ciudad de Bogotá (Colombia), Caracas (Venezuela) y Distrito Federal (México)" javierg@unam.mx

${ }^{3}$ Licenciado, maestro y doctor en Psicología por la Facultad de Psicología de la UNAM. Es Profesor Titular "C" en la FES Zaragoza, Docente en la UNAM durante 35 años. PRIDE nivel D. Tutor principal del doctorado en psicología y de las maestrías en psicología ambiental y trabajo social, de la UNAM y del doctorado en Educación en la Universidad INACE. Ha publicado más de 60 trabajos de investigación en revistas y capítulos en libros. Coeditó el libro Concepciones de la interacción social en el niño (2001 y 2006). Se encuentra en preparación de los libros Psicología ambiental: análisis de barreras para la sustentabilidad y Nuevas tendencias de la psicología de la conservación ambiental e Investigaciones psicoambientales en preescolares. Ha presentado más de cien ponencias en congresos mexicanos e internacionales. marcos.bustos@unam.mx
} 
retirement funds or economic supports. Empirical and testable lines of research are noted.

Keywords: Health, youth, old age, pension, discourse

\section{INTRODUCCIÓN}

Se estima que dos tercios de la población mundial están vinculados con los flujos migratorios. Una décima parte (115 millones) del total de la población de los países que integran la Organización para la Cooperación y el Desarrollo Económico (OCDE) son hijos de migrantes. Uno de cada tres estudiantes es descendiente de migrantes, $32 \%$ de la matrícula estudiantil lo conforman hijos de migrantes, pero el $75 \%$ de los migrantes hombres está en desempleo frente al 57\% de las mujeres desempleadas que son migrantes; sólo 4,3 millones de migrantes están legalmente estudiando o laborando y la inmigración crece un millón cada año (OCDE, 2010).

Durante el periodo que va de 2000 a 2012, Noruega lideró la recepción de migrantes, su tasa pasó del 0,6\% al 1,4\%; seguida de Alemania de 0,8\% a 1,2\% y de Australia de $0,5 \%$ a $1,1 \%$.

La Organización para la Cooperación y el Desarrollo Económico (OCDE), en su informe correspondiente a 2010, durante el periodo que va de 2000 a 2009 , estima que las muertes posteriores a los 30 días de ingreso al hospital pasaron de 8.3 a 4.1 en promedio por cada 100 casos para los países integrantes.

En los casos relacionados con asma, el promedio de la OCDE es de 51 casos por cada 100 mil habitantes mayores de 15 años. México ocupa el penúltimo sitio con 19 casos.

Por consiguiente, la problemática de salud ocupacional supone: 1) diferencias entre culturas dominantes y culturas migrantes; 2) el reflejo de tales diferencias en cuanto al servicio de salud en general y ocupacional en particular; 3) un sistema de adherencia al tratamiento que está en función de valores, creencias, actitudes, intenciones y comportamientos de profesionistas de la salud y del auto-cuidado; 4) la respuesta de los adultos mayores con respecto a su entorno económico, político, social y cultural que se refleja en el soporte familiar, la calidad de su atención y las políticas de salud.

La psicología de la salud en general y la psicología ocupacional en particular han establecido el Modelo de Demanda, Control y Soporte Social (MDCS) y el Modelo de 
Desbalance, Esfuerzo y Recompensa (MDER) para establecer los factores de riesgo cardiovascular, cerebro-vasculares y cardiopatía isquémica sobre los trastornos musculo-esquelético, estrés, ausentismo, accidentes, conflictos, insomnio, depresión y ansiedad.

La psicología organizacional ha estudiado los efectos de las demandas laborales sobre la salud ocupacional de trabajadores. A partir del Modelo de Demanda, Control y Soporte Social (MDCS) y el Modelo de Desbalance, Esfuerzo y Recompensa (MDER) ha establecido las relaciones de dependencia entre los factores de riesgo laboral y enfermedades, accidentes, conflictos y trastornos relacionados con el deterioro de la salud (Luceño, García, Rubio y Díaz, 2004).

El MDCS explica la relación entre el surgimiento del estrés con las demandas de la organización que son asimiladas como tareas excesivas por parte del trabajo con un nulo criterio de control de sus capacidades y esfuerzo. Una intensificación del ritmo laboral, exigencia de productividad, políticas contradictorias, conflictos, interrupciones determinan el auto-control; capacidades, habilidades, conocimientos, negociación del trabajador, así como accidentes o enfermedades (Fernández, Fernández y Siegrist, 2005).

El MDER advierte una relación asimétrica entre demandas y auto-control al considerar que las recompensas -rol, estatus, salario, reconocimiento- están en función de su esfuerzo, dedicación y productividad. El estrés emerge cuando la demanda, el esfuerzo y la recompensa son asimétricas (Gómez y Llanos, 2014).

En este sentido, los estudios psicológicos de la adherencia al tratamiento muestran que la condición minoritaria está asociada a una baja adherencia al tratamiento de enfermedades respiratorias en general y del asma en particular (Tao et al., 2008)

Bartlett et al., (2004) encontraron que la depresión por cuestiones económicas, sociales o afectivas en las mamás de niños migrantes con asma está asociada negativamente y significativamente al uso del inhalador y la ingesta del medicamento contra la enfermedad. Si bien la relación entre depresión materna y adherencia al tratamiento del asma no fue establecida directamente, sino sólo indirectamente a través de las creencias y actitudes, la condición de exclusión fue un factor determinante de la salud ocupacional que se diseminó en el uso del medicamento y dispositivo contra el asma. 
La adherencia al tratamiento al estar vinculada a estatus migratorios $\mathrm{y}$ depresiones emocionales está mediada por la consulta médica. Una frecuencia mayor de consultas explica la adherencia al tratamiento (Corsico et al., 2007).

Es decir que el estatus migratorio explica el manejo de un idioma diferente al de la cultura de origen, un uso deficiente de la lectura y la escritura, así como una disposición negativa a los derechos de servicios de salud y la adopción de estilos de vida saludables que inhiben la consulta médica y repercuten en la continuidad del tratamiento.

Kaptein et al., (2008) llevaron a cabo un metanalisis en torno a las percepciones de la enfermedad y encontraron que existe una tendencia desfavorable al uso de dispositivos -inhaladores- para el tratamiento del asma, sugieren que las intervenciones biomédicas y psicológicas al orientarse en el cambio de percepción de riesgo a utilidad de los medicamentos y dispositivos, incrementarán la adherencia al tratamiento.

En este sentido, la percepción de riesgo ha sido asociado al tipo de empleo que, en el caso de los migrantes, es más riesgoso que el de los oriundos, en consecuencia, la adherencia al tratamiento no sólo depende de la utilidad que la cultura dominante le atribuye a los medicamentos y dispositivos contra el asma, sino además a los atributos que la comunidad médica asocia con los valores y normas de migrantes.

Kardas, Lewel y Strzondala (2015) evidenciaron las diferencias culturales entre pacientes de asma al demostrar que la ignorancia y la desesperanza inciden sobre la adherencia al tratamiento. De este modo, el sistema de atención social al estar diseñado para el servicio de una cultura de derechos a la salud, excluye a las comunidades migrantes.

Sin embargo, Melton et al., (2014) encontraron una relación espuria entre la alfabetización médica y la adherencia al tratamiento. Además, plantean que el efecto de la consulta médica sobre la adherencia se llevó a cabo a partir de tres diferencias; a) información deseada e información recibida; b) ensayos de aciertos frente a ensayos de error; c) expectativas de pacientes y expectativas de médicos.

Los determinantes de la adherencia al tratamiento serían; 1) virtudes y estilos de vida, 2) tradiciones y valores culturales, 3) creencias en torno a información biomédica; 4) conocimientos de medicamentos y dispositivos; 5) normas sociales de aculturación, multiculturalismo o interculturales; 6) actitudes o disposiciones hacia el tratamiento del 
asma, medicamentos, dispositivos y profesionistas; 7) motivación al logro del tratamiento exitoso y 8) adherencia al tratamiento.

Sin embargo, la salud ocupacional también estaría influida por el entorno y el contexto político ya que, la contienda electoral está permeada de estímulos económicos relativos a la salud ocupacional mediante el otorgamiento de apoyos a los adultos mayores, becas a estudiantes, despensas a madres solteras que se suman a las promesas de campaña vinculadas al bienestar de los grupos vulnerables, marginados o excluidos.

En el caso de los adultos mayores estos han sido blanco de estrategias de captación de voto mediante el registro de casos en los que el Estado busca resarcir el pago de pensiones, fondos de retiro y ahorro. O bien, si se trata de casos de empleo informal, entonces el apoyo económico mensual a la tercera edad es otro instrumento de proselitismo electoral que se intensifica conforme la contienda se desarrolla y los comicios se aproximan (García, Carreón y Bustos, 2017).

Es así como la salud ocupacional se distancia de la adhesión al tratamiento, el soporte social, la recompensa laboral, el esfuerzo personal, la demanda familiar, el autocuidado y el autocontrol para establecer la regulación de estrategias mediáticas de captación de adherentes, simpatizantes y votantes de los partidos y candidatos involucrados en los comicios locales, estatales y federales.

En este escenario, las demandas políticas generan una intensificación de mensajes a favor o en contra de candidatos a la alcaldía, la presidencia o el senado. Se trata del establecimiento de temas tales como la salud, el bienestar y la calidad de vida de los adultos mayores con la finalidad de incidir en sus preferencias y decisiones de voto.

Sin embargo, la psicología social ha estudiado el fenómeno de proselitismo electoral sobre las audiencias, encontrando que la recepción de mensajes genera disposiciones en contra y a favor de candidatos. Es decir que los medios de comunicación generan una agenda con base en temas relacionados con la salud de grupos vulnerados, incidiendo en sus intenciones a través de emociones y en sus acciones mediante la desesperanza que supone la exclusión de los adultos mayores. En ese sentido, un sentimiento de indefensión es reorientado por las campañas políticas como una esperanza de vida al ser tomados en cuenta por un candidato o partido político como simpatizante y votante potencial (Carreón, 2016)). 
Tal fenómeno ha sido abordado desde el establecimiento de una agenda narrativa, posicionamiento evocativo y procesualismo simbólico como efectos de las campañas electorales en la soledad y desesperanza de adultos mayores con respecto a su salud personal y ocupacional (García, 2017).

El establecimiento de una agenda narrativa devela los temas de interés político que los medios de comunicación difunden con la intención de que los adultos mayores apoyen a un candidato político al opinar acerca de sus estrategias relativas a su bienestar personal, su salud y ocupación (García, Carreón y Hernández, 2017).

Una vez que se ha establecido la agenda de salud ocupacional, las campañas políticas generan posicionamientos discursivos que pueden ser observados en los discursos de los adultos mayores al recordar a las personas o grupos que les advertían sobre su empleo y ocupación en el futuro. En ese sentido, se trata de atribuciones de cuidado a personas, grupos e instituciones que los adultos mayores no sólo identifican y reconocen sino, además están dispuestos a apoyar.

Por último, el establecimiento de la agenda narrativa de salud ocupacional de los adultos mayores y el posicionamiento en contra o a favor de los mismos adultos mayores con respecto a quienes los cuidan genera un procesualismo simbólico. Se trata de una serie de opiniones negativas o positivas respecto a estrategias, instrumentos y sucesos que determinaron su actual situación económica y ocupacional (Carreón et al., 2017).

Si el establecimiento de una agenda narrativa incluye a la salud ocupacional como tema central para que los adultos mayores opinen al respecto y asocien este tema con la ayuda o apoyo económico que reciben directa o indirectamente por parte de sus gobernantes, repercutiendo en sus intenciones de voto, entonces el posicionamiento discursivo consistirá en atribuir soluciones a los candidatos, partidos o gobernantes que asocian con su situación ocupacional. De este modo, el procesualismo simbólico consistirá en cuestionar la relación entre las campañas políticas de salud ocupacional de candidatos con su situación económica y laboral.

El presente trabajo se propuso discutir los límites de los modelos psicológicos de salud ocupacional en la coyuntura electoral de una localidad del centro de México. Se realizó un estudio trasversal, exploratorio y cualitativo con una selección no probabilística de 7 migrantes de la tercera edad que acudieron a centros de salud de 
agosto 2015 a abril 2016 para consulta médica en las localidades de Huehuetoca, Teoloyucan y Coyotepec del Estado de México. Se encontró que las narrativas, los posicionamientos y los procesualismos giran en torno al Estado como administrador de fondos para el retiro.

Por consiguiente, la pregunta que guía la presente investigación es: ¿Cuáles son las oraciones discursivas que reflejan el establecimiento de una agenda ocupacional, un posicionamiento evocativo y un procesualismo simbólico en adultos mayores de diferentes estratos sociales, situacionales y ocupacionales del centro de México?

\section{MÉTODO}

Diseño. Se llevó a cabo un estudio no experimental, trasversal, exploratorio y cualitativo.

Muestra. Se realizó una selección no probabilística de 7 adultos mayores del Estado de México en las localidades de Huehuetoca, Teoloyucan y Coyotepec. Se consideró pertinente encuestar a quienes asistieron a los centros de salud durante el periodo que va de agosto de 2015 a abril de 2016 (véase Tabla 1).

\section{Tabla 1. Descriptivos de la muestra}

\begin{tabular}{llllll}
\hline Participante & Sexo & Edad & Estado civil & Escolaridad & Ingreso \\
\hline Adulto mayor empresario & Masculino & 66 & Casado & Licenciatura & 17000 pesos mensual \\
\hline Adulto mayor pensionado & Masculino & 71 & Casado & Licenciatura & 4600 pesos mensual \\
\hline Adulto mayor empleado & Femenino & 65 & Viudez & Preparatoria & 3000 pesos mensual \\
\hline Adulto mayor asilado & Femenino & 68 & Viudez & Preparatoria & Sin ingreso \\
\hline Adulto mayor desempleado & Femenino & 69 & Viudez & Secundaria & Sin ingreso \\
\hline Adulto mayor desocupado & Masculino & 65 & Separado & Secundaria & Sin ingreso \\
\hline Adulto mayor abandonado & Masculino & 66 & Separado & Primaria & Sin ingreso
\end{tabular}

Fuente: Elaborada con los datos del estudio

Instrumento. Se construyó una guía de entrevista con base en la literatura consultada la cual incluyó preguntas relativas a: 1) agenda narrativa, 2) posicionamiento evocativo y 3) procesualismo simbólico.

Agenda narrativa. Refiere a la influencia de temas establecidos en los medios de comunicación y su repercusión sobre la opinión de personas de referencia o grupos de pertenencia. 
Posicionamiento evocativo. Refiere a los recuerdos, anécdotas o artefactos asociados a la recepción de información y atribuidos a discursos de personas de referencia o grupos de pertenencia.

Procesualismo simbólico. Refiere a la necesidad, procesamiento, cuestionamiento y difusión de información relativa a la emancipación de o reivindicación de personas de referencias o grupos de pertenencia.

La guía de entrevistas incluyó preguntas tales como: ¿Cuál es el medio que utiliza para estar informado acerca de la salud, la juventud y la vejez? ¿Cuál es la información relacionada con la salud, la juventud y la vejez que más le llamó la atención en la última semana? ¿Cuál es el papel que los medios de comunicación le atribuyen al gobierno, los profesionistas -médicos, enfermeras, trabajadoras sociales, psicólogas-, su familia y a usted con respecto a la salud, la juventud y la vejez? ¿Cuáles son las estrategias que el gobierno debe seguir para que la salud de jóvenes y viejos mejore?

Se utilizaron matrices de análisis de contenido para vaciar y seleccionar extractos discursivos conforme a las categorías establecidas a fin de poder elaborar los esquemas discursivos (véanse Figuras 1, 2 y 3 ).

Procedimiento. Se utilizó la técnica Delphi para la homogenización de las palabras incluidas en los reactivos. Se garantizó el anonimato de las respuestas por escrito y se advirtió que los resultados del estudio no afectarían ni negativamente ni positivamente el estatus ambulatorio o la estancia de la persona entrevistada. Las entrevistas se realizaron en los centros de salud. La información se procesó en el Paquete de Análisis Cualitativo (QDA por su acrónimo en inglés, versión 4,0).

Análisis. A partir de las categorías de agenda narrativa, posicionamiento evocativo y procesualismo simbólico se analizaron los discursos de los entrevistados con respecto a la salud ocupacional y la contienda electoral. Se utilizó la técnica de síntomas para relacionar los extractos discursivos en torno a las tres categorías con la finalidad de inferir los significados de los extractos discursivos.

\section{RESULTADOS}

Los discursos de los adultos mayores entrevistados están centrados en las categorías de agenda narrativa, posicionamiento evocativo y procesualismo simbólico. 
En cada una de las categorías se incluye temas tales como: pensión, afore, seguro popular, gobierno, Internet, jubilación, vejez, trabajo, accidente, familia, educación, administración y juventud (véase Figuras 1, 2 y 3 ).

En el caso del establecimiento de la agenda narrativa está centrada en las pensiones, seguro popular y administración de ahorro para el retiro, pero si se considera la fuente del establecimiento de la agenda narrativa, entonces la prensa y la televisión están vinculadas a las opiniones de adultos mayores con un mayor estatus económico respecto a quienes están desempleados o desocupados y que su fuente de información es Internet y la radio (véase Figura 1).

Figura 1. Establecimiento de agenda narrativa en torno a la salud ocupacional como efecto de campañas electorales

Prensa

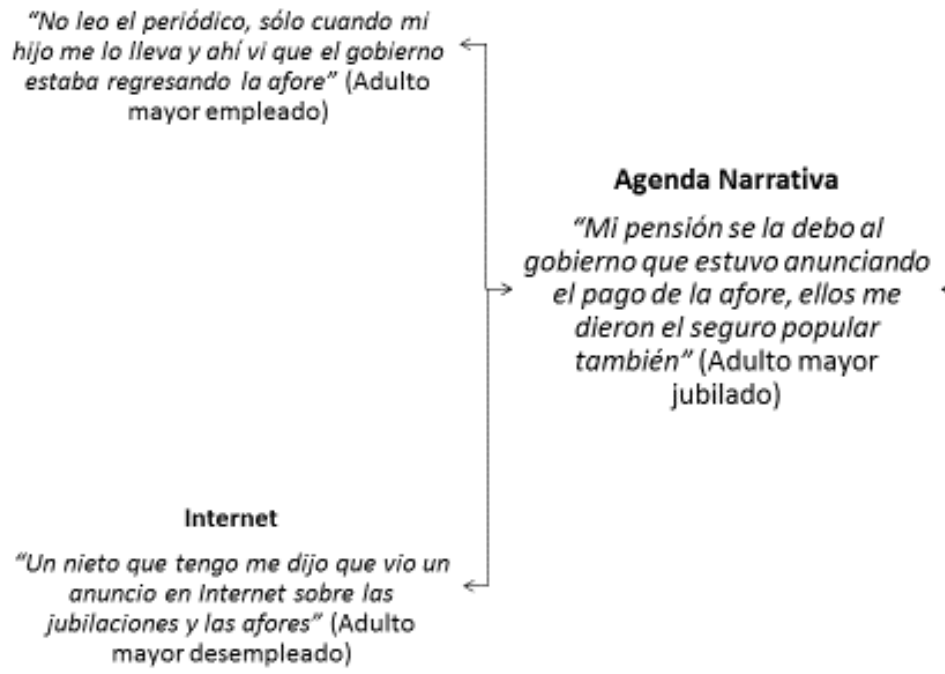

Televisión

"Vi hace poco que anunciaban el pago de la afore, el gobierno quiere ayudarnos a nosotros los viejos"

(Adulto mayor empresario)

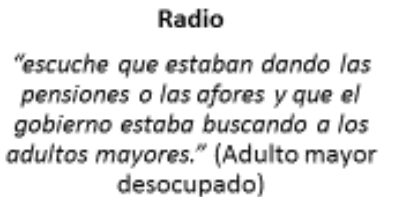

\section{Fuente: Elaborada con las entrevistas del estudio.}

Es decir que el establecimiento de la agenda en televisión y la prensa supone una audiencia con ingresos económicos que le permiten estar informados y al tener una creciente necedidad de información dependerían del establecimiento de una agenda centrada en las pensiones.

En el caso del posicionamiento evocativo al centrarse en el trabajo durante la juventud como una antecdente de la pensión, considerada una recompensa en la vejez, devela recuerdos y atribuciones de los entrevistados que estarían orientadas y dirigidas 
hacia la salud laboral en la juventud como antecedente de una salud ocupacional en la vejez (véase Figura 2).

Figura 2. Posicionamientos evocativos de los adultos maayores con respecto a su situación ocupacional

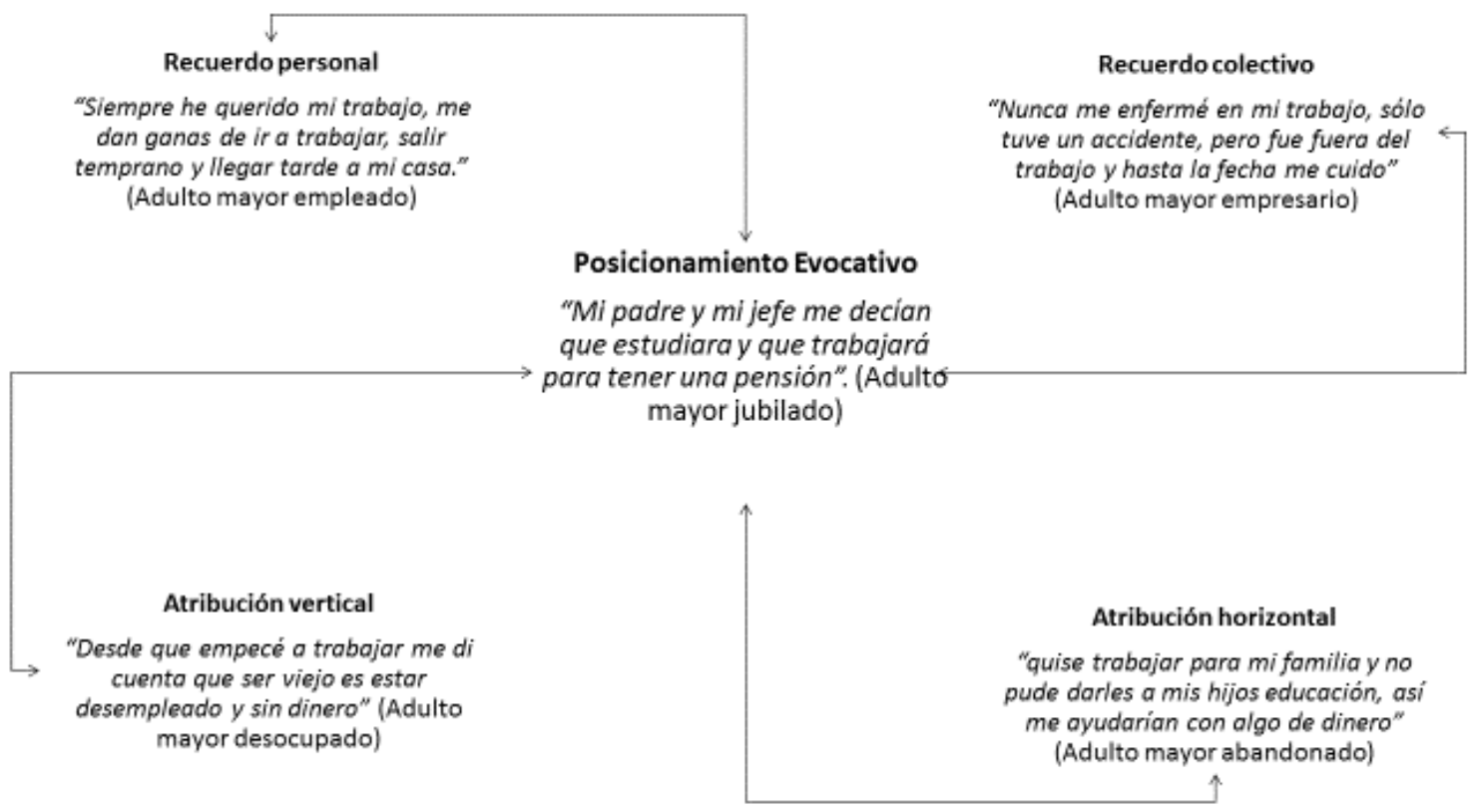

Fuente: Elaborada con las entrevistas del estudio.

El posicionamiento evocativo devela el vínculo estrecho entre la juventud y el trabajo y la vejez y la pensión, misma relación en la que el Estado jugaría un papel importante como administrador de los fondos de retiro, como compensador de quienes no tuvieron un empleo formal y como una fuente de ingreso para quienes están abandonados o no cuentan con un soporte familiar.

En el caso del procesualismo simbólico, las atribuciones de responsabilidad se dirigen hacia el gobierno, pero a la vez, existe un reconocimiento de que en la juventud, los adultos mayores entrevistados definieron su situación económica actual. Esto es así porque asumen que el Estado es un administrador de fondos de ahorro para el retiro, o bien, un gestor de apoyos a quienes no cuentan con el soporte familiar (véase Figura 3). 
Figura 3. Procesualismo simbólico de lso adultos mayores con respecto al Estado y su situación ocupacional

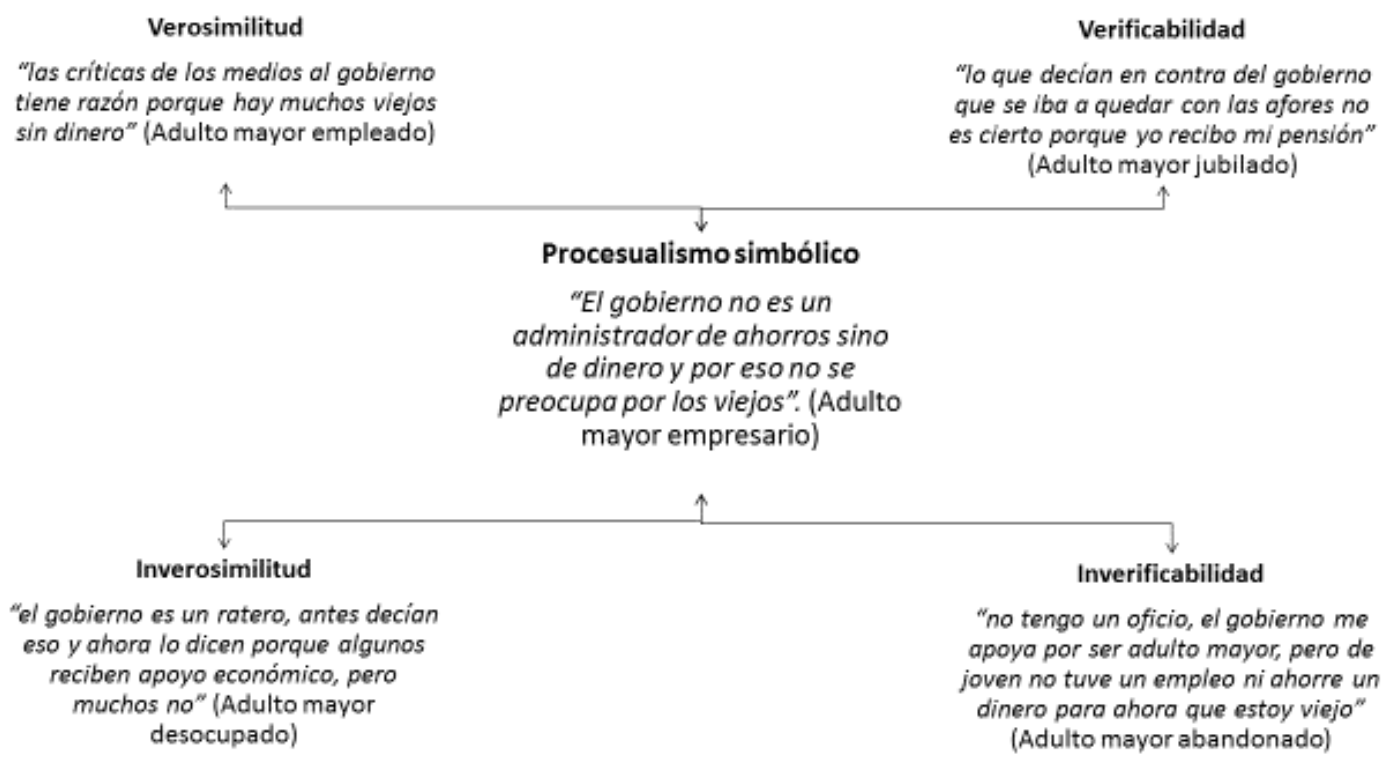

Fuente: Elaborada con las entrevistas del estudio.

En síntesis, los extractos discurisvos indican que existe un establecimiento de agenda centrada en la juventud y la vejez cuyas diferencias se observan en el trabajo formal que permite el otorgamiento de pensiones, gracias a la administración del Estado. O bien, el otorgamiento de apoyos, gracias a la beneficiencia del gobierno. Llama la atención que quienes tuvieron o cuentan con un empleo formal, apoyan la idea de que el Estado es un administrador de su dinero, pero en el caso de quienes han estado desempleados, desocupados o se sienten abandonados, el gobierno es un benefactor de su incapacidad de aprendizaje de un oficio o incapacidad de ahorro.

Es posible observar que los medios de comunicación han establecido una agenda que legitima la desesperanza de los adultos mayores al no haber aprovechado las oportunidades en su juventud, pero también legitima el apoyo a quienes no tuvieron un empleo formal que les asegurara un fondo de dinero para su vejez. En ambos casos, el Estado toma en cuenta a los adultos mayores que la sociedad excluye por el hecho de considerar que se trata de personas con capacidades, habilidades y conocimientos anacrónicos. 


\section{CONSIDERACIONES FINALES}

La salud ocupacional, en tanto resultado del aprendizaje de capacidades para el aprovechamiento de oportunidades de empleo y ahorro para el retiro, es un tema central en la agenda de las personas entrevistadas. Esa es la principal contribución del presente trabajo a la literatura especializada.

Sin embargo, la psicología que estudió el fenómeno de exclusión económica, política y social de los adultos mayores a través de los modelos de salud ocupacional, no ha explicado la prevención de enfermedades y accidentes, así como la adhesión al tratamiento o la rehabilitación de trabajadores accidentados o enfermos con respecto a su prospectiva de vejez, retiro y jubilación-pensión.

Los modelos que explican las asimetrías entre demandas y recursos, oportunidades y capacidades, requerimientos y esfuerzos, sanciones y recompensas en el ámbito ocupacional no consideran ni toman en cuenta siquiera la situación futura de los jóvenes que no tienen un trabajo estable y por consiguiente no ahorrarán para su retiro y manutención de su vejez.

En ese sentido, el presente trabajo ha evidenciado: 1) la salud ocupacional más allá de la organización o institución laboral, la juventud y la prevención de enfermedades y accidentes; 2) la relación estrecha entre la salud ocupacional con el retiro laboral, la pensión económica o el soporte familiar en la vejez; 3) el vínculo entre salud ocupacional con el contexto político electoral.

No obstante, los resultados del presente trabajo sólo se limitan a los siete adultos mayores entrevistados y las temáticas abordadas a través de la guía de entrevista y la matriz de análisis discursivo.

Por consiguiente, se recomienda: a) profundizar en las representaciones sociales de los adultos mayores con respecto a los temas de salud que se difunden en los medios de comunicación; b) contrastar las representaciones sociales con sus experiencias de apoyo a la tercera edad, pensiones y demás ingresos o prestaciones que el gobierno otorgue o difunda en la contienda electoral; c) elaborar un instrumento para medir las representaciones sociales de la salud ocupacional; d) establecer la confiabilidad y la validez del instrumento; e) contrastar el modelo de trayectorias de relaciones entre las categorías y variables relativas al establecimiento de agenda, el posicionamiento evocativo y el procesualismo simbólico. 


\section{REFERENCIAS}

Bartlett, S., Krishnan, J., Riekert, K., Butz, A., Malveaux, F. y Rand, C. (2004).Maternal depressive symptoms an adherence to therapy in inner city children wth asthma.Pediatrics, 113 (2), 229-237 |DOI: 10.1542/peds.113.2.229 J

Carreón, J. (2016). Desarrollo Humano: Gobernanza y Emprendimiento Social. México: UNAM-ENTS

Carreón, J., Hernández, J., Bustos, J. M. y García, C. (2017). Políticas de fomento empresarial y sus efectos sobre las percepciones de riesgo en caficultores de Xilitla, San Luis Potosí, centro de México. Poiesis, 32, 33-57

Corsico, A., Cazzoletti, L., Janson, R., Jarvis, D., Zola, M., Bugiani, M., Accordini, S., Villani, S., Marinoni, A., Gislason, D., Gulsvik, A., Pin, I., Vermeire, P. y Cerveri, I. (2007).Factors affecting adherence to asthma treatment in an international cohort of young and middle aged adults.Respiratory Medicinne, 101, 1363-1367 \{DOI: 10.1016/j.rmed.2006.11.012 ]

Fernández, A., Fernández, E. y Siegrist, J. (2005). El trabajo y sus repercusiones en la salud. El modelo desequilibrio-esfuerzo-recompensa. Calidad Asistencial, 20 (3), $165-170$

García, C. (2017). Los estudios psicológicos del establecimiento de agenda. Especificación y discusión de un modelo de identidad conformista versus cooperativista. Nomadas, 50, 1-18

García, C., Carreón, J. y Bustos, J. M. (2017). Los estudios de la migración laboral: estructura factorial exploratoria del estigma laboral. Eureka, 14 (1), 1-16

García, C., Carreón, J. y Hernández, J. (2017). Límites de los modelos de salud ocupacional. estudio de adhesión al tratamiento del asma en trabajadores migrantes adultos mayores del Estado de México. Visión Gerencial, 16 (1), 103118

Gómez, V. y Llanos, A. (2014). Factores psicosociales del origen laboral, estrés y morbilidad, en el mundo. Psicología desde el Caribe, 31 (2) 354-385

Kaptein, A., Hughes, B., Sharloo, M., Fischer, M., Sinoel, L., Weiman, J. y Rabe, K. (2008). Illness perception about asthma are determinants of outcome. Journal of Asthma, 45, 459-464 [DOI: 10.1080/02770900802040043 ]

Kardas, P., Lewel, P. y Strzondala, M. (2015). Adherence to tratament in asthma and COPD patients in their doctor assessment.Pneumologia I AlergiaPolska, 83 (6), 436-444 [DOI: : 10.5603/PiAP.2015.0072 ]

Luceño, L., García, J., Rubio, S. y Díaz, E. (2004). Factores psicosociales en el entorno laboral, estrés y enfermedad. Edupsyche, 3 (1), 95-108

Melton, C., Graff, C., Norlin, G., Brown, L. y Bailed, J. (2014). Health literacy an asthma management among African-American adults: an interpretative phenomenological analysis. Journal of Asthma, 51 (7), 703-713 |DOI: 10.3109/02770903.2014.906605 J 
Tao, L., Bilderback, A., Bender, B.,Wambolt, F., Turner, C., Rand, S. y Bartlett, S. (2008). Do asthma medication beliefs mediate the relationships between minority status and adherence to therapy? Journal of Asthma, 45, 33-37 |DOI: $10.1080 / 02770900701815552$ J 